\title{
Passive Phloem Loading and Long-Distance Transport in a Synthetic Tree-on-a-Chip
}

\author{
by \\ Jean Comtet
}

Submitted to the Department of Mechanical Engineering in partial fulfillment of the requirements for the degree of

Master of Science in Mechanical Engineering at the

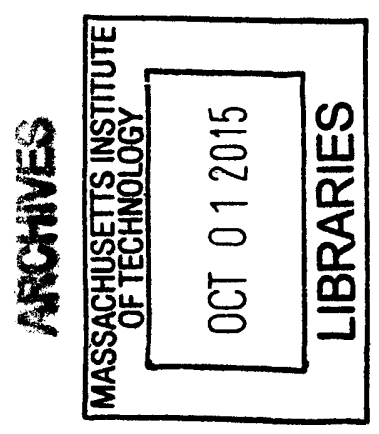

\section{MASSACHUSETTS INSTITUTE OF TECHNOLOGY}

September 2015

(C) Massachusetts Institute of Technology 2015. All rights reserved.

Author

\section{Signature redacted}

Department of Mechanical Engineering

July 30,2015

Certified by

\section{Signature redacted}

Accepted by

Anette E. Hosoi

Professor, Mechanical Engineering

Thesis Supervisor

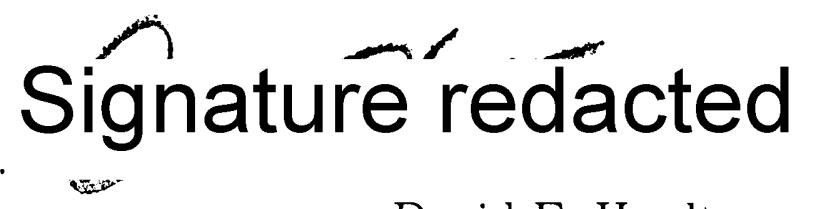

David E. Hardt Chairman, Department Committee on Graduate Theses 


\title{
Passive Phloem Loading and Long-Distance Transport in a Synthetic Tree-on-a-Chip
}

by

\author{
Jean Comtet
}

\author{
Submitted to the Department of Mechanical Engineering \\ on July 30, 2015, in partial fulfillment of the \\ requirements for the degree of \\ Master of Science in Mechanical Engineering
}

\begin{abstract}
According to the Münch mechanism, vascular plants rely on osmotic pressure gradients to export sugars from regions of synthesis (mature leaves) to sugar sinks (roots, fruits). A crucial step in this process is the loading of sugars from photosynthetic cells to the export conduit (the phloem). In this thesis, we developed a synthetic microfluidic osmotic pump mimicking the mechanism of passive phloem loading, where sugars are transported by diffusion from a sugar reservoir to the phloem.

This design allows the development of steady flow over several hours. We show that in our system, phloem concentration is set by a relative balance between loading by diffusion from the source and export by convection through the phloem, via a single nondimensional system-scale Peclet number that we call the flushing number. For large flushing numbers, export is limited by diffusion from the source, and flow rates scale weakly with transport resistance. For low flushing numbers, export is limited by convection through the phloem and phloem concentration is close to that of the source, leading to efficient export of water and sugars.

In plants, passive phloem loading is used predominantly by trees. We show that the hydrostatic pressures developed in our synthetic system can reach up to ten bars and are thus compatible with the pressures expected to drive long-distance transport in large trees. Moreover, we show that the regime of efficient export in passive loaders is more accessible to plants with large transport resistances, providing a rational for the use of the passive loading mechanism by most tree species.
\end{abstract}

Thesis Supervisor: Anette E. Hosoi

Title: Professor, Mechanical Engineering 


\section{Acknowledgments}

First and foremost, I would like to thank my advisor, Peko Hosoi for giving me a lot of freedom and allowing me to develop and carry out my personal research interests. Thank you Peko for being so receptive, encouraging and giving me invaluable support.

This work would not have been possible without my initial interaction with Abe Stroock and Bob Turgeon during a summer research internship at Cornell. In particular, I am very grateful to Abe for his advice and for teaching me so many things. During the development of this work, I also benefited from very fruitful interactions with Kaare Jensen; it has been a pleasure to work with him.

Back at MIT, I would like to thank all the people from HML for creating a nice working environment. In particular, I am extremely gratefull to José for his invaluable support, advice and sharing his personal experience of science. I would also like to thank Bavand for being so knowledgeable and for his great experimental advice and suggestions, as well as Alex and Ahmed. Finally, I wish to thank all the people with whom I have had the pleasure to interact with on a regular basis at HML.

I have also had the pleasure to work on a side project with John Bush during my time at MIT. It was a very valuable experience and I really enjoyed working with him.

Finally, I wish to deeply thank Emmanuelle, my girlfriend, for her commitment and supporting me throughout this work. 


\section{Contents}

$\begin{array}{lll}1 & \text { Introduction } & 13\end{array}$

2 Sap flow in a synthetic passive phloem loader $\quad 17$

3 Flushing number and transport regimes $\quad 25$

4 Phloem hydrostatic pressure and energy conversion 29

5 Phloem loading strategies in trees and herbaceous plants $\quad 33$

6 Discussion $\quad 37$

A Solution to the coupled transport equations $\quad 41$

B Evalutation of membrane resistances and dextran viscosity 43 


\section{List of Figures}

1-1 Schematic of a tree, where xylem (in blue) pulls water to the leaves via evaporation (blue arrows), and phloem (in red) exports the photosynthesized sugars (pink arrows) from sources (light green) to sinks. Synthesized in the mesophyll cells, sugars are first transported to the phloem (red line), where the large sugar concentration drives via osmosis a water flow from the adjacent xylem tissues (blue line), creating a bulk flow through the phloem conduit (downward red arrow). . . .

2-1 Simplified model for water and sugar transport in passive loaders and in our synthetic osmotic pump. Sugars (red dots), stored in the source (concentration $c_{0}$, green) diffuse to the phloem (concentration $c$, light red) through a porous wall of permeability $K_{\mathrm{D}}\left[\mathrm{m}^{3} / \mathrm{s}\right]$, where they drive, by osmosis through a semipermeable membrane of hydraulic resistance $R_{\mathrm{M}}\left[\mathrm{Pa} . \mathrm{s} / \mathrm{m}^{3}\right]$, a flow of water from the xylem (light blue). Water and sugars are subsequently convected to the roots through the transport phloem of resistance $R_{\mathrm{T}}\left[\mathrm{Pa} . \mathrm{s} / \mathrm{m}^{3}\right]$. . . . . . . . . . . . .

2-2 Cross-sectional view of the osmotic pump. The phloem channel $(\mathrm{P})$ is in contact with the xylem channel $(\mathrm{X})$ via a semipermeable membrane (blue), and with the source $(\mathrm{S})$ via a physical membrane (red). The pump inlet brings pure water to the xylem channel (X). A hydraulic resistance mimicking a hydraulic load can be placed at the outlet of the phloem channel $(\mathrm{P}) . \ldots \ldots \ldots$ 
2-3 Flow rates are monitored by connecting the pump inlet and outlet to two partially glass-filled capillaries (upper left inset). Red curve: Evolution of the flow rate with time for a design without any source (schematic in red), where the phloem is initially filled with a solution of concentration $c$. The flow rate decreases with time, due to depletion of the phloem concentration. Blue curve: Evolution of the flow rate with time for a design including source, at a concentration $c_{0}$, connected to the phloem, initially at a concentration $c=0$ (schematic in blue). The flow rate reaches a steady-state value where sugar loading via diffusion and sugar export via convection balance. . . . . . . . . . .

2-4 Photograph of the device. The blue arrow indicates xylem water inlet, connected to the xylem channel (blue dashed box). The source channel is filled with a sugar concentration $c_{0}$ at the beginning of an experiment (green dashed box). The phloem channel is between the source and xylem channel (not visible on the photograph). A solution of water and sugars flows out of the phloem channel (pink arrow) through a capillary tube of adjustable transport resistance $R_{\mathrm{T}}$. The pressures developing in the phloem channel can be directly measured using a pressure sensor (white box) . . . . . . . . . . . .

2-5 Steady-state flow rate as a function of source concentration $c_{0}$, for different values of the semipermeable membrane resistances $R_{M}$ and porous wall mass transfer coefficient $K_{D}$. Red symbols: $R_{M}=4040$ Pa.s/nL, green symbols: $R_{M}=18000$ Pa.s $/ \mathrm{nL}$. Dots: $K_{D}=12 \mathrm{~nL} / \mathrm{s}$. Triangles: $K_{D}=0.44 \mathrm{~nL} / \mathrm{s}$. Dotted lines are fits to equation (5), allowing the determination of the mass transfer coefficients $K_{\mathrm{D}}$. Plain lines represent the expected flow rate for the classical Münch model where phloem concentration $c$ equal to source concentration $c_{0} \ldots \ldots$ 
3-1 For a fixed membrane permeability and source concentration, the flushing number decreases with increasing hydraulic resistance and leads to a build-up of phloem concentration. (A) For a low flushing number (physical membrane permeability large compared to the mean flow rate), the export is limited by water convection through the hydraulic network. Phloem concentration is approximately equal to source concentration $\left(c \sim c_{0}\right)$, and water and sugar export are equal to their maximal value $Q_{\max }$ and $\phi_{\max }$ for the given hydraulic resistance. (B) Intermediary flushing number, where $c \leq c_{0}$. (C) For a large flushing number (physical membrane permeability small compared to the mean flow rate), the export is limited by diffusion through the physical membrane. The concentration in the phloem is small compared to the one in the source $\left(c \approx c_{0} / \sqrt{f}\right)$ and water and sugar export are small compared to their maximal value. . . . . . . . . . . . . .

3-2 Variation of the dimensionless flow rate $Q / K_{\mathrm{D}}$ with the flushing number. The experimental points correspond to the data points of Fig. 2-5. Inset : Variation of the dimensionless flow rate $Q / Q_{\max }$ and dimensionless concentration $c / c_{0}$ with flushing number $f \ldots \ldots \ldots$

4-1 Variation of flow rate (A), phloem hydrostatic pressure (B), mechanical power density (C) and phloem concentration (D) with transport resistance $R_{\mathrm{T}}$ for a fixed source concentration $c_{0}=110 \mathrm{mmol} / \mathrm{L}$, membrane resistance $R_{\mathrm{M}}=18000 \mathrm{~Pa} . \mathrm{s} / \mathrm{nL}$ and a physical membrane of permeability $K_{D}=15 \mathrm{~nL} / \mathrm{s}$, estimated when the phloem channel is not pressurized. The plain lines correspond to the theoretical expressions from Eq. 4.1 and Eq. 3.2 with $K_{D}=15 \mathrm{~nL} / \mathrm{s}$. Dotted lines correspond to similar expressions with $K_{D}=7 \mathrm{~nL} / \mathrm{s}$. . . . . . . . 
5-1 Increasing flushing numbers lead to a decrease in sugar export rate relative to the maximal export $\phi_{\mathrm{M}}=R T c_{0}^{2} / R_{\text {tot }}$. Histogram : estimation of the flushing number $L_{P}\left(R T c_{0}-\rho g h\right) / k_{\mathrm{D}}\left(1+R_{\mathrm{T}} / R_{\mathrm{M}}\right)^{-1}$ with $R_{\mathrm{T}}$ the transport phloem resistance and $R_{M}$ the loading phloem membrane resistance, $-\rho g h$ the xylem pressure decreasing with plant height $h$ and $R T c_{0}$ the osmotic pressure in the source, for a sample of Gymnosperm trees and herbaceous plants. . . . . . . . . . . . . 


\section{Chapter 1}

\section{Introduction}

Sugars, photosynthesized in the mesophyll of plant leaves, are conveyed by a viscous sap to regions of growth or storage (such as roots or fruits) through a specialized microfluidic vascular network called the phloem [17]. In this network, sugars play a dual role, acting both as an energy carrier and a motile force generator, where the osmotic pressure of the phloem sap provides the necessary force to drive a convective flow from sugar sources to sinks. Despite consensus over the basic principles allowing sugar transport in plants, the complexity of phloem physiology and its coupling with other tissues has made a quantitative understanding of transport challenging, and it is still unclear whether the pressures necessary to drive long distance transport in plants can be osmotically powered.

The mechanism by which plants transport sugars from the mesophyll (Fig. 11; green) to export conduits (Fig. 1-1; red), known as phloem loading, is crucial. Loading lowers the water potential of phloem cells in the leaf to levels necessary to import water via osmosis from the adjacent xylem tissues (Fig. 1-1; blue), setting the pressure head available to power long-distance transport. In most plants, energy is used to increase the sugar concentration in leaf phloem to levels higher than those in mesophyll cells. Because transport resistances scale with the height of the plant, one might expect trees to require the largest phloem pressures of all plants in order to sustain comparable rates of sugar export. Oddly, the majority of trees seem to rely on a passive phloem loading mechanism where the sugar concentration in the 


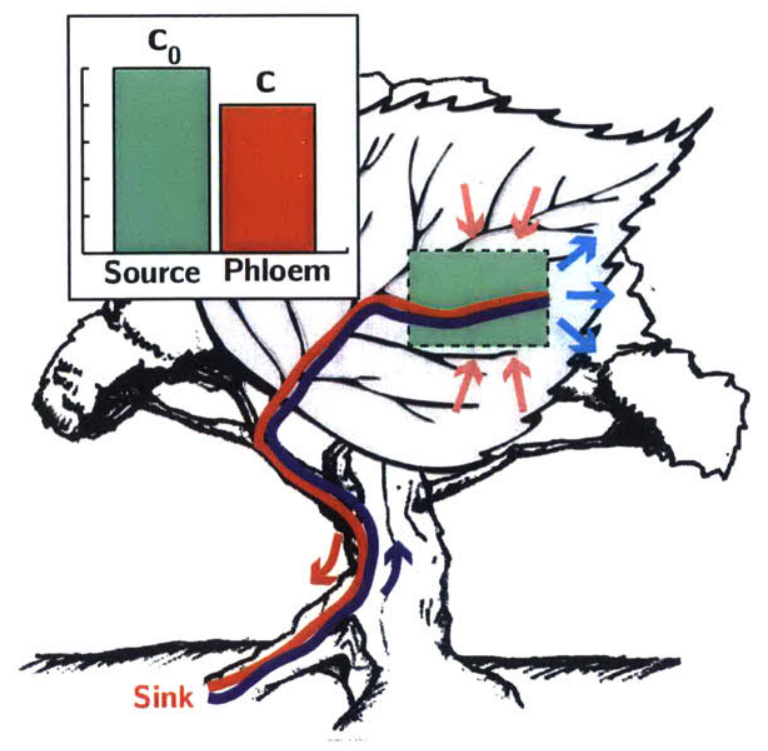

Figure 1-1: Schematic of a tree, where xylem (in blue) pulls water to the leaves via evaporation (blue arrows), and phloem (in red) exports the photosynthesized sugars (pink arrows) from sources (light green) to sinks. Synthesized in the mesophyll cells, sugars are first transported to the phloem (red line), where the large sugar concentration drives via osmosis a water flow from the adjacent xylem tissues (blue line), creating a bulk flow through the phloem conduit (downward red arrow).

phloem is slightly lower than that of the mesophyll (Fig. 1-1; diagram), thus reducing the apparent available driving force [18]. Experiments have provided evidence for the use of passive loading in trees $[19,21]$. However the ability of the passive loading mechanism to generate long distance transport in plants and the absence of active loading in trees is not well understood [18]. For example, Fu et al. suggested that the low water potential in leaf xylem of trees requires large sugar concentrations in the mesophylls, making active phloem loading unnecessary [4]. This factor however, is not directly coupled to the kinetics of phloem transport.

To explore whether passive phloem loading can generate the pressures necessary to drive sugar translocation in large trees and generate long distance transport, and to determine why trees might favor this mechanism, we investigate transport dynamics in a synthetic microfluidic system in which sugars are metered via passive loading - a so-called tree-on-a-chip. Experimental systems designed to mimic transport processes in plants have been used by several authors to test mechanistic hypotheses of vascular 
physiology $[2,14,3,13,20,10,7]$. To demonstrate his theory of osmotically generated pressure flows in plants, Ernst Münch used an artificial system consisting of two semipermeable reservoirs in contact with a water bath, each filled with different sugar concentrations and connected by a glass tube. He observed a convective flow of water from the large to the low concentration reservoir. More refined experiments were carried out later by Eschrich, Evert, and Young [3], and by Lang [13], who verified quantitatively the speed of translocation. Recently, Jensen et al. reproduced those experiments in a microfluidic device, at the length-scales relevant in plants $[10,7]$. Existing synthetic designs, however, are limited by the fact that the solutes present in the source are depleted by convection over time, preventing steady flows and pressures over experimentally relevant timescales. Moreover, phloem concentration in these systems are set at the beginning of the experiments and do not reproduce the dynamical interaction of the phloem tube with the photosynthetic cells and the xylem.

To overcome these challenges, we designed a biomimetic model of a passive phloem loader that allowed us to replicate the dynamically determined parameters of passive loading mechanism under conditions that range from small herbs to large trees. Due to the presence of a sugar source reservoir, this bioinspired osmotic pump can run at steady-state for several hours, providing a new strategy for pumping microscale flows in lab-on-a-chip applications, and allowing precise measurements of the physicochemical coupling at the system scale. The driving force of the system is the osmotic pressure in the phloem channel, which is proportional to the local solute concentration c. We first show that this concentration is dynamically determined by an interplay between the diffusive loading from the source and advective export through the phloem. We find that this coupling is well-described by a non-dimensional ratio of timescales associated with advective and diffusive processes (similar to a Peclet number) that we dub the flushing number $f$. When the flushing number is large, export is limited by the diffusion of solutes from the source to the phloem, and phloem concentration is much smaller than the concentration of the source. In this regime, the flow rate and sugar export scales weakly with the hydraulic resistance, providing a novel en- 
gineering solution to situations where constant export of water or solutes is needed regardless of the outlet pressure or system's resistance. This large- $f$ regime would be characteristic of small herbaceous plants, where the resistance to flow in the stem is so low that sugars which diffuse into the phloem would be quickly swept away. In contrast, when the flushing number is small, our synthetic device reveals that transport is constrained by the convective export of solutes through the hydraulic circuit. In this limit, the concentration in the phloem is close to that of the source, leading to efficient export of solutes and water. Low values of the flushing number $f$ would be found in trees, and we find that hydrostatic pressures which can be developed in our synthetic system are comparable with the pressure expected to drive translocation in large trees. We thus argue that active phloem loading is not required for long distance transport in plants, and that passive loaders can develop pressure heads that are comparable to active loaders, at the cost of large sugar concentration in their leaves. Moreover, we show that for passive loaders, efficient export is more easily accessible to plants with large transport resistances and low xylem pressures like trees. Along with previous biological experiments rationalizing the use of passive loading by large trees [4], our synthetic experiments show that passive loading can optimally generate long distance transport in large plants and thus provides a rationale for the use of passive loading via diffusion that is observed in most trees species. 


\section{Chapter 2}

\section{Sap flow in a synthetic passive}

\section{phloem loader}

Plants distribute sugars synthesized in the mesophyll cells by a bulk liquid flow through the phloem conduits, the sieve tubes (Figs. 1-1; 2-1). In passive loaders, sugars (Fig. 2-1, red dots) first diffuse from the mesophyll (light green) to the conductive phloem sieve elements (pink) through small plasmodesmata pores. The presence of sugars in the phloem lowers the chemical potential of the sap, drawing in water from the adjacent xylem tissue (light blue). This in turn drives a bulk flow of water and sugars out of the leaf through the transport phloem (red and blue downward arrows).

Using microfluidic technology, we built an osmotic micropump that captures the main physical ingredients of sugar translocation in a passive phloem loader (Fig. 2-2). The phloem channel, connected to the pump outlet (light red) is in contact with a large sugar source (mesophyll, light green) via a porous wall (red) that allows sugars to diffuse from the source chamber to the phloem, at a rate proportional to the concentration difference. The phloem channel is also in contact with the xylem water source (light blue) via a semipermeable membrane (blue) that allows for osmotic exchange of water between the two channels.

We first flush the entire device with pure water, then, we introduce a dextran solution (here dextran plays the role of sugar in a live plant) of concentration $c_{0}$ to the mesophyll source reservoir. To measure the flow rate exiting the synthetic phloem, 


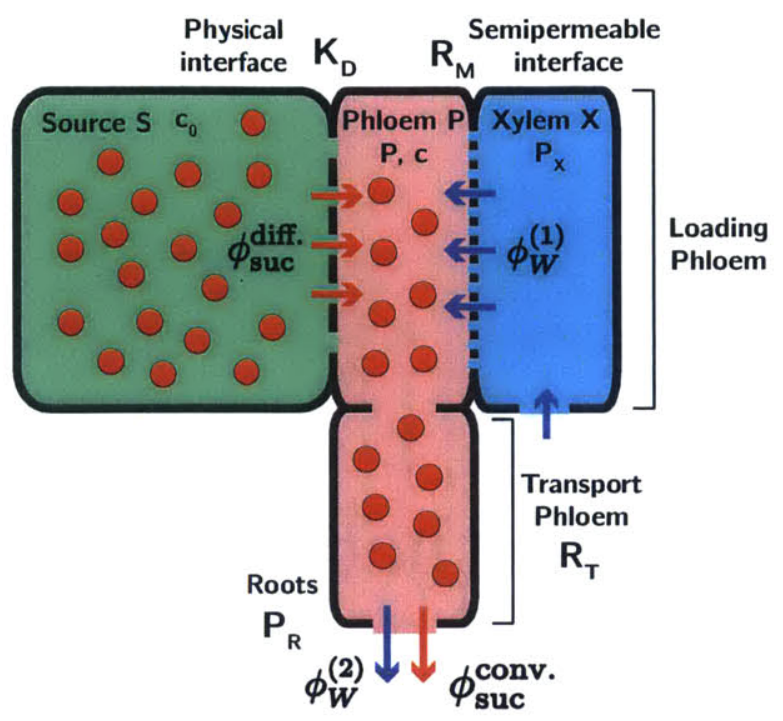

Figure 2-1: Simplified model for water and sugar transport in passive loaders and in our synthetic osmotic pump. Sugars (red dots), stored in the source (concentration $c_{0}$, green) diffuse to the phloem (concentration $c$, light red) through a porous wall of permeability $K_{\mathrm{D}}\left[\mathrm{m}^{3} / \mathrm{s}\right]$, where they drive, by osmosis through a semipermeable membrane of hydraulic resistance $R_{\mathrm{M}}\left[\mathrm{Pa} . \mathrm{s} / \mathrm{m}^{3}\right]$, a flow of water from the xylem (light blue). Water and sugars are subsequently convected to the roots through the transport phloem of resistance $R_{\mathrm{T}}\left[\mathrm{Pa} . \mathrm{s} / \mathrm{m}^{3}\right]$.

the inlet and outlet of the device are connected to partially filled glass capillary tubes (Fig. 2-3, inset). Tracking the meniscus position over time allows us to measure the flow rate $Q(t)$ (Fig. 2-3). Initially a transient phase is observed in which the flow rate increases with time due to a gradual buildup of sugars in phloem. Following this transient, stable osmotic pumping is observed (Fig. 2-3, blue line).

The steady state is characterized by a balance between diffusive transport of sugars from the mesophyll to the phloem (which tends to increase the concentration in the phloem) and osmotic flow of water from the xylem to the sink (which sweeps away sugars and reduces the concentration in the phloem). Our device thus exhibits a qualitatively different behavior than that observed in previous experiments $[14,3$, $13,10,7]$, where solutes present in the source are depleted over time preventing the creation of steady flow over experimental timescales (Fig.2-3, red line). In contrast, our device captures essential aspects of the dynamic interaction that is thought to exist between the phloem tube, the photosynthetic cells and the xylem, and produces a 


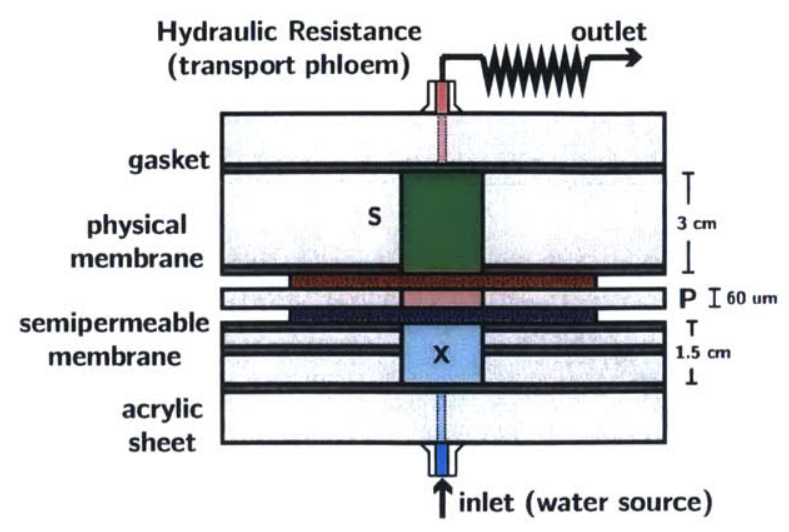

Figure 2-2: Cross-sectional view of the osmotic pump. The phloem channel $(\mathrm{P})$ is in contact with the xylem channel $(\mathrm{X})$ via a semipermeable membrane (blue), and with the source $(\mathrm{S})$ via a physical membrane (red). The pump inlet brings pure water to the xylem channel (X). A hydraulic resistance mimicking a hydraulic load can be placed at the outlet of the phloem channel $(\mathrm{P})$.

steady flow state where the output flow rate and phloem concentration is dynamically determined by the hydraulic and transport connectivity between the different cells.

The microfluidic osmotic pump is made by sandwiching together the gaskets, channels and dialysis membranes (Fig. 2-4). All channels are $1 \mathrm{~mm}$ wide. The sugar source and the part of the phloem channel interacting with the sugar and water source is $52 \mathrm{~mm}$ long. The phloem channel is $77.5 \mathrm{~mm}$ long and the xylem channel is 104 mm long. Gaskets were craft-cut (using a Graphtec CE-6000 Vinyl Cutter) out of 100 um thick soft Polyvinylchloride (PVC) films. The phloem channel was craft-cut out of $60 \mathrm{um}$ thick rigid Polyester (PET) sheets. The water and sugar source channels were laser-cut out of $1 \mathrm{~mm}$ thick acrylic sheets, then sanded and polished (Novus Plastic). The device was screwed together between the aluminium plates. Flexible capillary tubes were connected to the channels using nanoports (Idex H\&S).

We use $6 \mathrm{kDa}$ Dextran (Alfa Aesar) as the solute. To obtain physical and semipermeable membranes, we used Cellulose Ester (CE) dialysis membranes (SpectrumLabs) of various Molecular Weight Cut-Off (MWCO). For MWCO larger than $6 \mathrm{kDa}$, the membrane will be permeable to dextran, whereas for MWCO smaller than 6 $\mathrm{kDA}$, the membrane is considered semipermeable, i.e. reflects dextran but lets water go through. We used semipermeable membranes of respective MWCO $0.1 \mathrm{kDa}, 1$ 


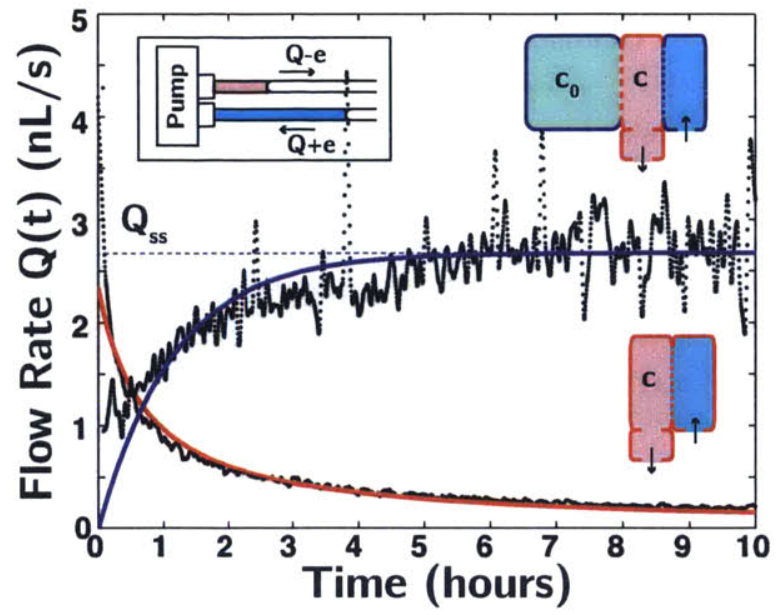

Figure 2-3: Flow rates are monitored by connecting the pump inlet and outlet to two partially glass-filled capillaries (upper left inset). Red curve: Evolution of the flow rate with time for a design without any source (schematic in red), where the phloem is initially filled with a solution of concentration $c$. The flow rate decreases with time, due to depletion of the phloem concentration. Blue curve: Evolution of the flow rate with time for a design including source, at a concentration $c_{0}$, connected to the phloem, initially at a concentration $c=0$ (schematic in blue). The flow rate reaches a steady-state value where sugar loading via diffusion and sugar export via convection balance.

$\mathrm{kDa}$ and 3.5-5 kDa, and physical membranes of MWCO 8-10 kDa.

Flow rates were measured by connecting the inlet and outlet of the pump to two partially filled glass capillaries of diameters $500 \mu \mathrm{m}$ and $800 \mu \mathrm{m}$. For a quasi static meniscus, the capillary pressure due to the water meniscus in the glass capillary is the same in the inlet and outlet of the pump. Monitoring the evolution of the meniscus position over time by time-lapse photography allowed us to calculate the flow rates $Q_{\text {inlet }}$ and $Q_{\text {outlet }}$ (Cf. Fig. 2-4). Kymographs for meniscus position over time were obtained using ImageJ. A measurement was considered valid when the absolute difference between inlet and outlet flow rates differed by less than $0.5 \mathrm{~nL} / \mathrm{s}$. This difference was attributed to the evaporative flux of water $Q_{\text {evap }}$ at the two menisci interface. The pump flow rate was then estimated as $Q_{\text {pump }}=\left(Q_{\text {inlet }}+Q_{\text {outlet }}\right) / 2$.

Due to the large size of the channel reservoir (around $16 \mathrm{~cm}^{3}, 50$ times larger than the phloem channel), the source concentration did not vary much over the time of the experiments, and steady flow rate could be observed for several hours. 


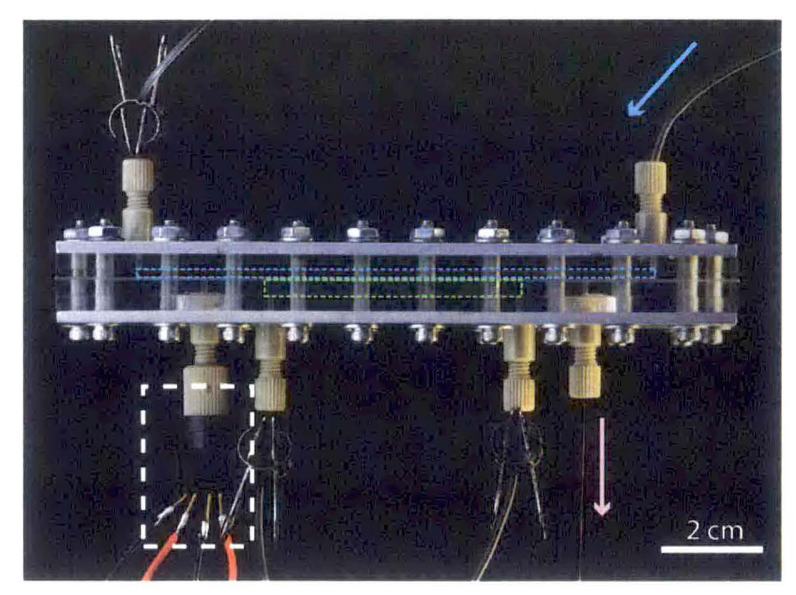

Figure 2-4: Photograph of the device. The blue arrow indicates xylem water inlet, connected to the xylem channel (blue dashed box). The source channel is filled with a sugar concentration $c_{0}$ at the beginning of an experiment (green dashed box). The phloem channel is between the source and xylem channel (not visible on the photograph). A solution of water and sugars flows out of the phloem channel (pink arrow) through a capillary tube of adjustable transport resistance $R_{\mathrm{T}}$. The pressures developing in the phloem channel can be directly measured using a pressure sensor (white box).

When the MWCO of the semipermeable membrane was close to the Dextran size (semipermeable membrane of 3.5-5 kDa MWCO) and the concentration in the phloem channel large was large, we observed a decrease in water flow approximately one hour after the establishment of a steady flow. This decrease was attributed to the diffusive leakage of solute to the xylem channel through the semipermeable membrane.

To identify the parameters that influence sugar transport in a passive phloem loader, we systematically varied the source concentration $c_{0}\left[\mathrm{~mol} / \mathrm{m}^{3}\right]$, the loading conductance of the mesophyll-sieve element interface (characterized by a diffusive mass transfer coefficient $K_{\mathrm{D}}\left[\mathrm{m}^{3} / \mathrm{s}\right]$ ), the hydraulic semipermeable membrane resistance $R_{\mathrm{M}}$ and the hydraulic transport resistance $R_{\mathrm{T}}\left[\mathrm{Pa} . \mathrm{s} / \mathrm{m}^{3}\right]$. We denote the total hydraulic resistance of the system $R_{\mathrm{tot}}=R_{\mathrm{M}}+R_{\mathrm{T}}$. The hydraulic resistances of the semipermeable membranes were measured independently, by applying a solution of known concentration on one side of the membrane and measuring the resulting flow rate.

Fig. 2-5 shows that the flow rate $Q$ increases with the mesophyll source concen- 
tration $c_{0}$, and with the mesophyll-to-phloem loading conductance $K_{\mathrm{D}}$ (red triangles and dots, Fig. 2-5) but decreases with increasing membrane hydraulic resistance (red and green dots, Fig. 2-5). The dotted line represents the flow rates that are expected when the phloem concentration is equal to the source concentration $\left(c=c_{0}\right)$, as predicted by the classical models of Münch transport, for which $Q \sim R T c_{0} / R_{\text {tot }}$. We observed that the flow rates can deviate significantly from this prediction, since the finite physical permeability $K_{\mathrm{D}}$ introduces a depletion of the phloem concentration relative to that of the source. Mass transfer coefficient were estimated by fitting Eq. 3.3 to the points of Fig. 2-5.

To estimate the deviation from ideal Münch flow, we develop a mathematical model of passive phloem loading. The sugar concentration $c\left[\mathrm{~mol} / \mathrm{m}^{3}\right]$ in the phloem chamber (of volume $16 \mathrm{~cm}^{3}$ ) increases due to diffusion from the mesophyll and decreases by convective flow out of the phloem according to the conservation equation: $d c / d t=K_{\mathrm{D}}\left(c_{0}-c\right)-Q c$. The first term on right-hand side represents the diffusive transport of solutes across the porous barrier between mesophyll and phloem (proportional to the concentration difference between the two cells), and the second term represents the advective flow of solutes out of the phloem. When the system has reached a steady-state (Fig.2-3, red curve), there is a balance between diffusion and advection

$$
K_{\mathrm{D}}\left(c_{0}-c\right)=Q c
$$

The flow rate $\mathrm{Q}$ is determined by the water potential gradient between phloem and xylem, and by the pressure gradient between the phloem and the sink.

$$
\begin{aligned}
R_{\mathrm{M}} Q & =P_{X}-P_{P}+R T c \\
R_{\mathrm{T}} Q & =P_{P}-P_{R}
\end{aligned}
$$

where $P_{X}, P_{P}$ and $P_{R}$ are respectively xylem, phloem, and root pressures, $R$ is the ideal gas constant, $T$ the temperature and $R T c$ is the osmotic pressure in the phloem, 


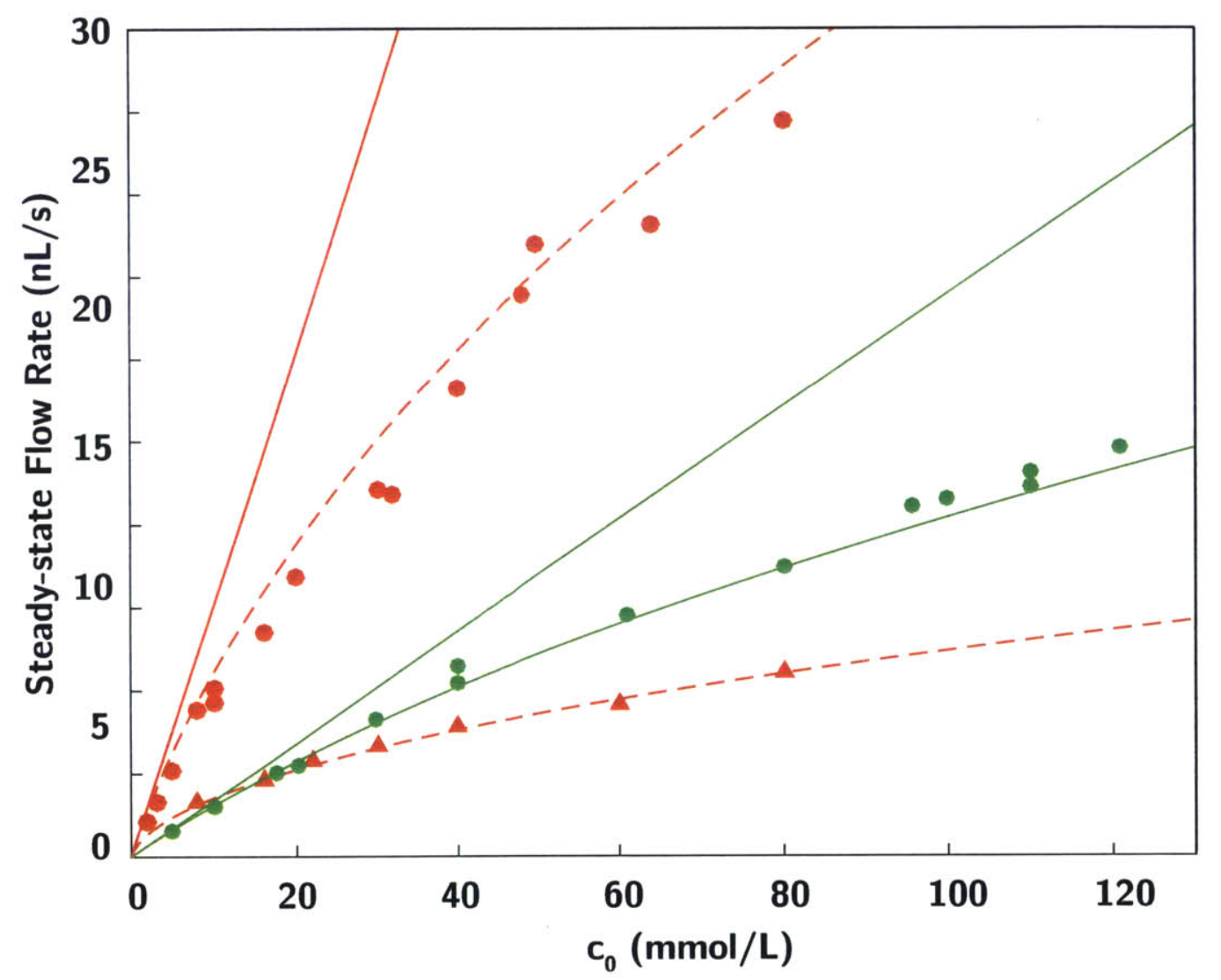

Figure 2-5: Steady-state flow rate as a function of source concentration $c_{0}$, for different values of the semipermeable membrane resistances $R_{M}$ and porous wall mass transfer coefficient $K_{D}$. Red symbols: $R_{M}=4040$ Pa.s $/ \mathrm{nL}$, green symbols: $R_{M}=18000$ Pa.s $/ \mathrm{nL}$. Dots: $K_{D}=12 \mathrm{~nL} / \mathrm{s}$. Triangles: $K_{D}=0.44 \mathrm{~nL} / \mathrm{s}$. Dotted lines are fits to equation (5), allowing the determination of the mass transfer coefficients $K_{\mathrm{D}}$. Plain lines represent the expected flow rate for the classical Münch model where phloem concentration $c$ equal to source concentration $c_{0}$. 
proportional to solute concentration $c$ (an osmotic coefficient $\alpha$ characterizing solute non-ideality can be taken into account in the expression of the osmotic pressure and the hydraulic resistance. We find $\alpha=4.7$ for dextran, and include this factor in the values of the membrane resistances. See Appendix B for details). Summing these two equations allows us to express $Q$ as a sole function of $P_{X}, P_{R}$ and $c$.

$$
\left(R_{\mathrm{M}}+R_{\mathrm{T}}\right) Q=P_{X}-P_{R}+R T c
$$

From Eqns. 2.1 and 2.4, we can express the steady state flow rate $Q$ and sugar concentration in the phloem $c$ as a function of the device parameters, as shown in Fig. 2-5 (Appendix A). 


\section{Chapter 3}

\section{Flushing number and transport regimes}

The steady state of our system is characterized by a balance between diffusive transport from the mesophyll to the phloem (which tends to increase the concentration in the phloem) and osmotic flow of water from the xylem (which sweeps away sugars and dilutes the phloem). We are thus able to represent the system behavior by a single "flushing number" $f$ that characterizes the relative importance of these two fluxes.

$$
f=\frac{\text { ADVECTION }}{\text { DIFFUSION }}=\frac{R T c_{0}+P_{\mathrm{X}}-P_{R}}{K_{\mathrm{D}_{\bullet}} R_{\mathrm{tot}}}=\frac{Q_{\max }}{K_{\mathrm{D}}}
$$

where $Q_{\max }=\left(R T c_{0}+P_{\mathrm{X}}-P_{\mathrm{P}}\right) / R_{\text {tot }}$ is the flow rate obtained in ideal Münch flow. Note that the flushing number is analogous to a system-scale (as opposed to a local) Peclet number. To simplify the subsequent analysis, we make the assumption that the external pressure gradient is small $\Delta P=P_{R}-P_{\mathrm{X}} \ll R_{\mathrm{tot}} K_{D}$. This assumption is valid both in our experiments (where $\Delta P$ is strictly null) and in the majority of plants (see Chap. 5). The dimensionless flow rate $Q$ and the concentration $c$ can be expressed in terms of the flushing number $f$ using Eqns. (2.1-2.4)

$$
\frac{Q}{Q_{\max }}=\frac{c}{c_{0}}=\frac{\sqrt{1+4 f}-1}{2 f}
$$


A

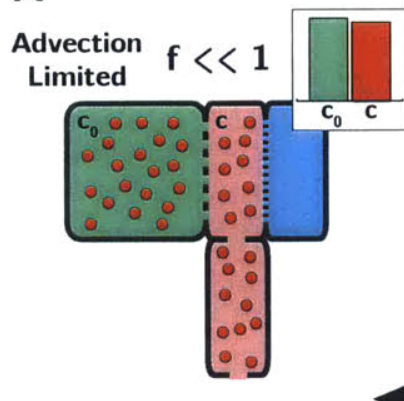

B

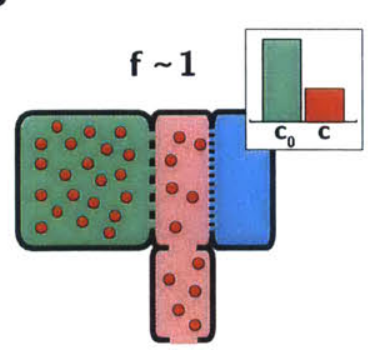

increasing hydraulic resistance
C

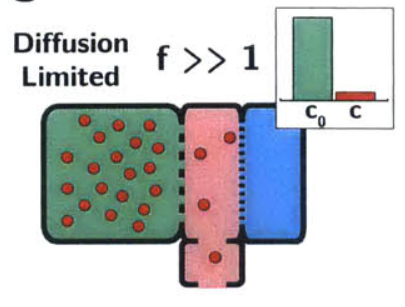

Figure 3-1: For a fixed membrane permeability and source concentration, the flushing number decreases with increasing hydraulic resistance and leads to a build-up of phloem concentration. (A) For a low flushing number (physical membrane permeability large compared to the mean flow rate), the export is limited by water convection through the hydraulic network. Phloem concentration is approximately equal to source concentration $\left(c \sim c_{0}\right)$, and water and sugar export are equal to their maximal value $Q_{\max }$ and $\phi_{\max }$ for the given hydraulic resistance. (B) Intermediary flushing number, where $c \leq c_{0}$. (C) For a large flushing number (physical membrane permeability small compared to the mean flow rate), the export is limited by diffusion through the physical membrane. The concentration in the phloem is small compared to the one in the source $\left(c \approx c_{0} / \sqrt{f}\right)$ and water and sugar export are small compared to their maximal value.

Alternatively, the flow rate $Q$ can be non-dimensionalized by the loading mass transfer coefficient $K_{\mathrm{D}}$.

$$
\frac{Q}{K_{\mathrm{D}}}=\frac{\sqrt{1+4 f}-1}{2}
$$

Flow rates and concentration measured in the synthetic device (Fig. 2-5) collapse onto a single curve when plotted according to Eq. (3.3) (Fig. 3-2). The sugar export rate can be written as the product of flow rate and concentration and nondimensionalized by $Q_{\max }$ and $c_{0}$ (Fig. 5-1):

$$
\frac{\phi}{\phi_{\max }}=\frac{Q c}{Q_{\max } c_{0}}=\frac{(\sqrt{1+4 f}-1)^{2}}{4 f^{2}}
$$

Hence the transport efficiency depends on the magnitude of the flushing number (Eq. (3.4), Fig. 3-2 inset and 5-1). For low flushing numbers $(f \ll 1)$, the membrane mass 
transfer coefficient is large compared to the mean flow rate (i.e. the total hydraulic resistance is large) and the diffusion of solute through the porous wall is fast compared to convection out of the phloem (Fig. 3-1). This leads to similar concentrations in the phloem and mesophyll and ideal Münch pumping

$$
c \approx c_{0}, Q \approx \frac{R T c_{0}}{R_{\text {tot }}} \text { and } \phi \approx \frac{R T c_{0}^{2}}{R_{\text {tot }}} \text { for } f \ll 1
$$

In this regime, the export is limited only by the convection of water through the hydraulic circuit and the export of both sugar and water is optimal, since the concentration in the phloem is close to that of the source. For large flushing numbers $(f \gg 1)$, where the membrane mass transfer coefficient is small compared to the mean flow rate (i.e. the total hydraulic resistance is small) the diffusion of solute through the physical membrane is slow compared to convection out of the phloem (Fig. 31C). In this case, the residence time of solute in the phloem is short compared to the diffusion time through the physical membrane. The concentration $c$ in the phloem is thus much smaller than the concentration in the source. Expanding equation (3.2), we find

$$
c \approx c_{0} / \sqrt{f}=\sqrt{R_{\mathrm{tot}} K_{\mathrm{D}} c_{0} / R T} \text { and } Q=\frac{Q_{\max }}{\sqrt{f}}=\sqrt{K_{\mathrm{D}} R T c_{0} / R_{\mathrm{tot}}} \text { for } f \gg 1
$$

In this regime, the export is limited by the diffusion of solutes through the physical membrane and the water flow rate shows a weak, $-1 / 2$ power scaling with the system hydraulic resistance (Fig. 3-2; eq. (3.6)). In addition, the total export of sugars is

$$
\phi=\frac{\phi_{\max }}{f}=K_{\mathrm{D}} c_{0} \text { for } f \gg 1
$$

which corresponds to the diffusive transport across the porous interface. The scalings of flow rate $Q \sim R_{\mathrm{tot}}^{-1 / 2}$ and sugar concentration $c \sim R_{\mathrm{tot}}^{1 / 2}$ with the hydraulic resistance $R_{\text {tot }}$ in the diffusion-limited regime $(f \gg 1)$ are surprising and reflect an adaptation of the pump driving force to the system's demand. In fact, in the diffusion-limited regime, an increase of the system's hydraulic resistance will lead to a build-up of 


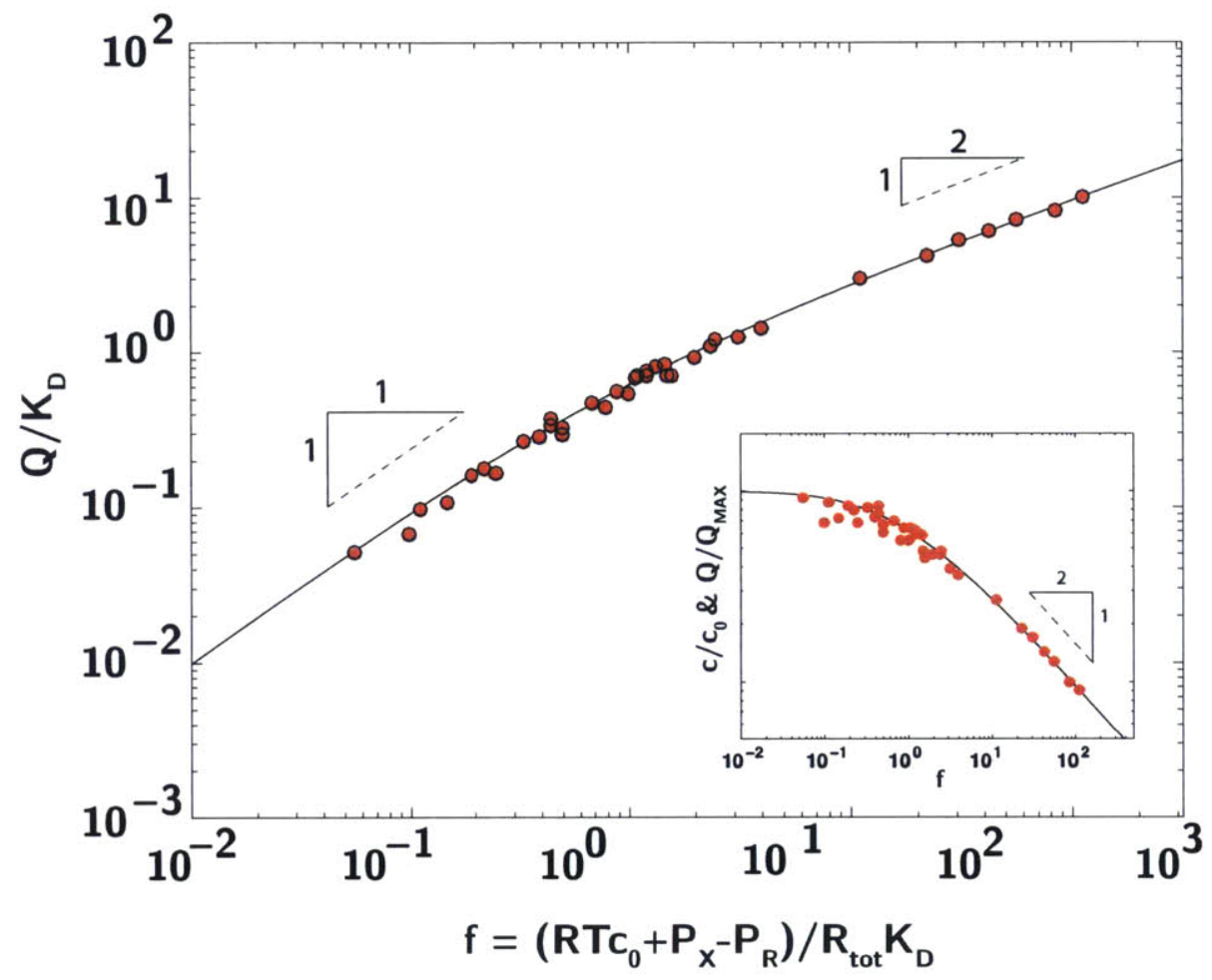

Figure 3-2: Variation of the dimensionless flow rate $Q / K_{\mathrm{D}}$ with the flushing number. The experimental points correspond to the data points of Fig. 2-5. Inset : Variation of the dimensionless flow rate $Q / Q_{\max }$ and dimensionless concentration $c / c_{0}$ with flushing number $f$.

the concentration $c$ in the phloem (Figs. 3-1B-C). Such regimes present potential applications in situation where steady flow is necessary, regardless of the output resistance or backpressure. 


\section{Chapter 4}

\section{Phloem hydrostatic pressure and}

\section{energy conversion}

Passive phloem loading has long been thought to be insufficient to drive transport over long distances, because the downhill concentration gradient between the mesophyll and the phloem reduces the available driving pressure $\Delta P \sim R T c$, where $c$ is the concentration in the phloem conduits [18]. To test this conjecture, we connect the device outlet to capillary tubes (Polymicro Technology) of radius 10 and $15 \mu \mathrm{m}$ and length ranging from 5 to $40 \mathrm{~cm}$ (Fig. 2-2) and measure the resulting flow rate $Q$ and hydrostatic pressure $\Delta P$ developped in the phloem for fixed membrane resistance, loading conductance and a source concentration of $110 \mathrm{mmol} / \mathrm{L}$ corresponding to an osmotic pressure of 12.7 bars. Pressures are directly measured using two pressure sensors of respective range 0 to 100 psi and 0 to 250 psi (Honeywell 26PCFFM6G and 26PCGFM6G), which can be directly screwed to one end of the phloem channel (Fig. 2-4).

At the beginning of a pressure measurement experiment, we flush the capillary tube, phloem and xylem with pure water and fill the source with a fixed concentration $c_{0}=110 \mathrm{mmol} / \mathrm{L}$. Due to the build-up of pressure, the phloem channel dilates and we observe initially a larger flow rate in the inlet than in the outlet. We then let the pump run for several hours such that it reaches a steady state where phloem and capillary tubes are filled with a dextran solution of fixed concentration $c$, and the 

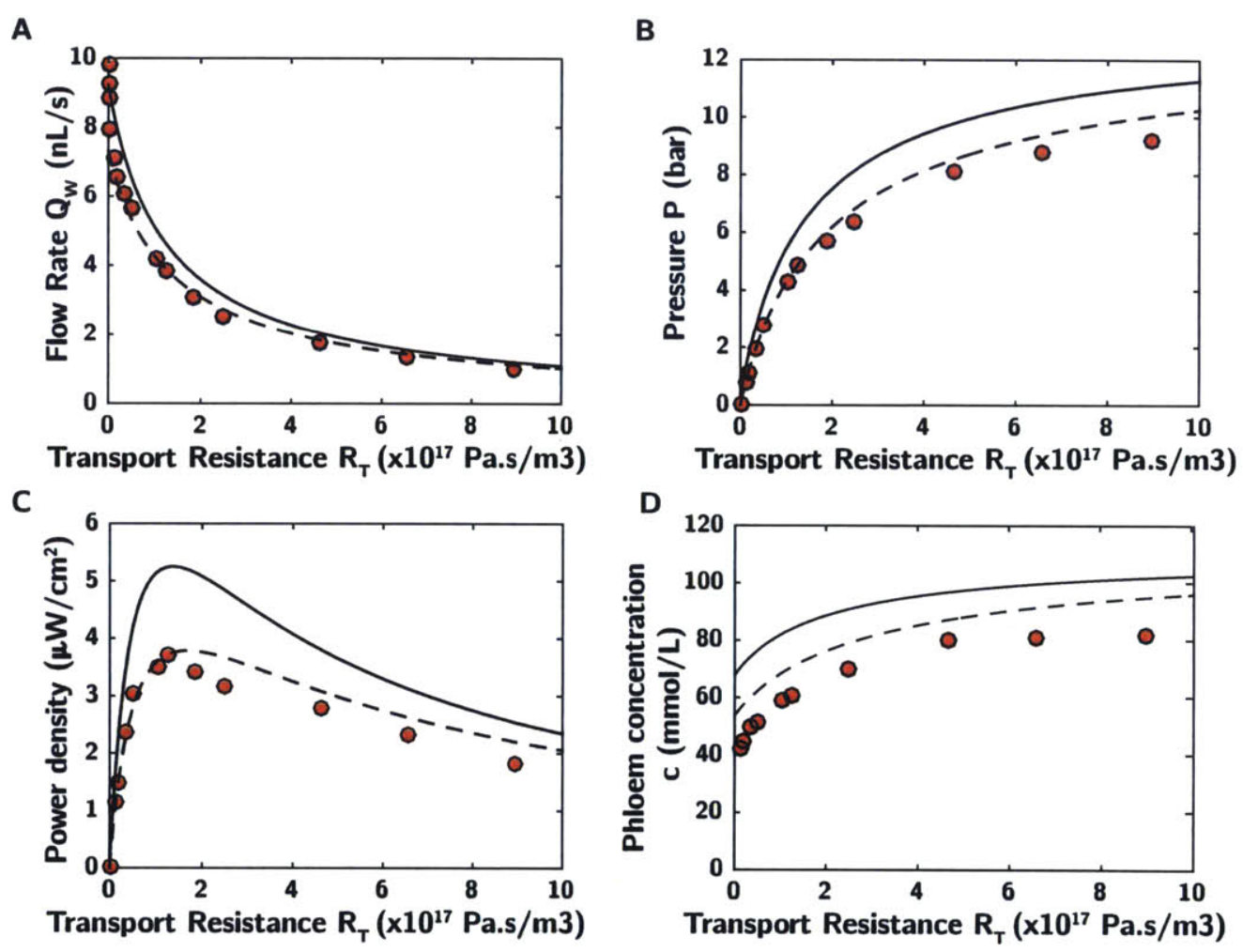

Figure 4-1: Variation of flow rate (A), phloem hydrostatic pressure (B), mechanical power density (C) and phloem concentration (D) with transport resistance $R_{\mathrm{T}}$ for a fixed source concentration $c_{0}=110 \mathrm{mmol} / \mathrm{L}$, membrane resistance $R_{\mathrm{M}}=18000$ Pa.s/nL and a physical membrane of permeability $K_{D}=15 \mathrm{~nL} / \mathrm{s}$, estimated when the phloem channel is not pressurized. The plain lines correspond to the theoretical expressions from Eq. 4.1 and Eq. 3.2 with $K_{D}=15 \mathrm{~nL} / \mathrm{s}$. Dotted lines correspond to similar expressions with $K_{D}=7 \mathrm{~nL} / \mathrm{s}$. 
transport resistance $R_{\mathrm{T}}$ remains constant. Once this steady state has been reached, we flush the xylem channel with pure water, refill the sugar reservoir, and carry out the experiment again. This time, pressure builds up more rapidly, as the capillary tube is already filled with the viscous dextran solution.

We estimate the transport resistance at steady state as $R_{\mathrm{T}}=\Delta P / Q . R_{\mathrm{T}}$ can reach values as high as $10^{18} \mathrm{~Pa} . \mathrm{s} / \mathrm{m}^{3}$, equivalent to a continuous sieve tube of radius $20 \mu \mathrm{m}$ and up to 10 meters in length. As the transport resistance is increased, we observe a decrease in the pumping speed (Fig. 4-1A) and an increase in the hydrostatic pressure (Fig. 4-1B). Hydrostatic pressures in the phloem of our synthetic passive phloem loader reach values up to 10 bars, of the same order than the pressures needed to drive translocation in large trees [18].

The total hydraulic power developed by the pump can be expressed as $\Pi=Q \Delta P$. We plot in Fig. 4-1C the pump mechanical power density, in $\mu \mathrm{W}$ per area of membrane. For low flushing number, mechanical power is maximized for equal values of the membrane and transport resistances $R_{\mathrm{M}} \approx R_{\mathrm{T}}$, a relation well documented in a broad range of plants [11].

To estimate the concentration of the dextran solution flowing in the capillary tubes (Fig. 4-1D), we measure the variation of dextran viscosity with concentration (Appendix B) and estimate the viscosity of the solution flowing in the tube by comparing the value of the hydraulic resistance $R_{\mathrm{T}}=\Delta P / Q$ with the expected poiseuille resistance calculated from the length and radius of the capillary tubes. As expected from Fig. 3-1, phloem concentration increases with $R_{\mathrm{T}}$, due to a decrease in the flushing number.

We compare those experimental results with our model. The variation of flow rate and phloem pressure with $R_{\mathrm{T}}$ is given by

$$
Q=\frac{K_{D}}{2} \sqrt{1+\frac{4 \alpha R T c_{0}}{K_{D}\left(\alpha R_{M}+R_{\mathrm{T}}\right)}} \text { and } \Delta P=\frac{R_{\mathrm{T}} K_{D}}{2} \sqrt{1+\frac{4 \alpha R T c_{0}}{K_{D}\left(\alpha R_{M}+R_{\mathrm{T}}\right)}}
$$

where we have introduced dextran osmotic coefficient $\alpha=4.7$.

The plain lines in Fig. 4-1 show the expected variation of flow rate, pressure, 
power density and concentration, using the value of $K_{D}=14.9 \mathrm{~nL} / \mathrm{s}$ obtained from the best fit of the data of Fig. 2-5, when the phloem channel is not pressurized. This value of the mass transfer coefficient leads to a slight overestimation of all variables. In fact, the dilatation of the phloem channel due to the build-up of pressure leads to a decrease in the effective mass transfer coefficient of dextran. Taking $K_{D}=7 \mathrm{~nL} / \mathrm{s}$ gives a better agreement between our model and experiments. 


\section{Chapter 5}

\section{Phloem loading strategies in trees and herbaceous plants}

Our experiments have shown the existence of two distinct transport regimes in our synthetic passive phloem loader (Fig. 3-1). We now seek to determine which transport regimes plants occupy, and how this regime depends on macroscopic plants traits such as plant height $h$, sieve tube radius $a$ and plasmodesmatal interface permeability. To estimate the flushing number, we take the pressure differential between xylem and root as scaling with the hydrostatic pressure $\rho g h$ with $\rho$ water density and $g$ gravitational constant. We can rewrite the flushing number as:

$$
f=\frac{\left[R T c_{0}-\rho g h\right] L_{p}}{k_{D}} \frac{1}{1+R_{\mathrm{T}} / R_{\mathrm{M}}}
$$

where we have introduced the membrane permeability $L_{p}$ and diffusive conductivity $k_{D}$, which are related to the membrane resistance $R_{M}=1 /\left(A L_{p}\right)$ and diffusive conductance $K_{D}=A k_{D}$ by the mesophyll-to-phloem contact area A. The first factor $\left(R T c_{0}-\rho g h\right) L_{p} / k_{D}$ only depends on the available osmotic pressure in the mesophyll and on the permeability ratio, whereas the second factor depends only on the ratio of the transport resistance and semipermeable membrane resistances $R_{\mathrm{T}} / R_{\mathrm{M}}$. Passive loaders can thus reach the regime of low flushing number by increasing the permeability of their plasmodesmatal interface, by the presence of large transport resistances 
or by offsetting the osmotic driving force by low negative xylem pressure.

Several factors limit the permeability of the mesophyll to phloem plasmodesmatal interface. First, plasmodesmatal pores allow small sugar molecules to diffuse, while maintaining the integrity of the mesophyll cells by preventing larger structural proteins or organelles from leaving the cell. We thus expect plasmodesmatal pore radii to be close to the sucrose hydrodynamic radius. Second, the density of plasmodesmata at the mesophyll/phloem interface is also limited by the mechanical stability of the interface which constrains the maximum density of plasmodesmatal pores admissible. The density of plasmodesmata at this interface is known to vary considerably among species [6] [5]. However, for symplastic loaders (type 1 and 1-2a in Gamalei's classification [6]), this density is close to a maximal value of 10 and 40 plasmodesmata $/ \mu \mathrm{m}^{2}$ of interface, suggesting that the permeability $k_{D}$ of the plasmodesmatal interface does not vary dramatically among symplastic loaders.

We estimate $k_{D}$ as $N \rho_{\mathrm{p}} \pi r^{2} D / d$ with $d \approx 0.25 \mu \mathrm{m}$ the typical length of a plasmodesmata, $\rho_{\mathrm{p}} \approx 40 / \mu \mathrm{m}^{2}$ the density of plasmodesmata, $D$ the effective diffusion coefficient of sucrose through the pores, $N=9$ the number of pores per plasmodesmata and $r \approx 1 \mathrm{~nm}$ the radius of one pore. Assuming hindered sucrose transport [1], we obtain $D \approx 4.7 \cdot 10^{-11} \mathrm{~m}^{2} / \mathrm{s}$, leading to $k_{D} \approx 2.1 \cdot 10^{-7} \mathrm{~m} / \mathrm{s}$. We further assume $L_{P} \approx 5 \cdot 10^{-14} \mathrm{~m} / \mathrm{s} / \mathrm{Pa}[11]$ and $R T c_{0} \approx 10$ bar, leading to $R T c_{0} L_{P} / k_{D} \approx 0.25$.

Based on these considerations, we compute in Fig. 5-1 the flushing number $f$ for a set of angiosperm plants that are thought to load passively (trees) and actively (herbaceous plants). To estimate the value of the relative membrane and transport resistances in plants, we approximate source length $l$ by the length of the leaf, and transport length $h$ by the height of the plant. For cylindrical sieve elements of radius $a$, we find, following Jensen et al. $[11,9] R_{M} / R_{T}=16 \eta L_{P} h l / a^{3}$.

To estimate the flushing number in herbaceous plants, we use the dataset assembled by Jensen et al. [11], containing informations on sieve tube radius $a$, leaf length $l$ and plant height $h$ on $N=15$ herbaceous species. For angiosperm trees, we use another dataset assembled by Jensen et al. [8], which contains information only on $h$ and $l$. However, because the sieve tube radius of angiosperm trees appears to be 


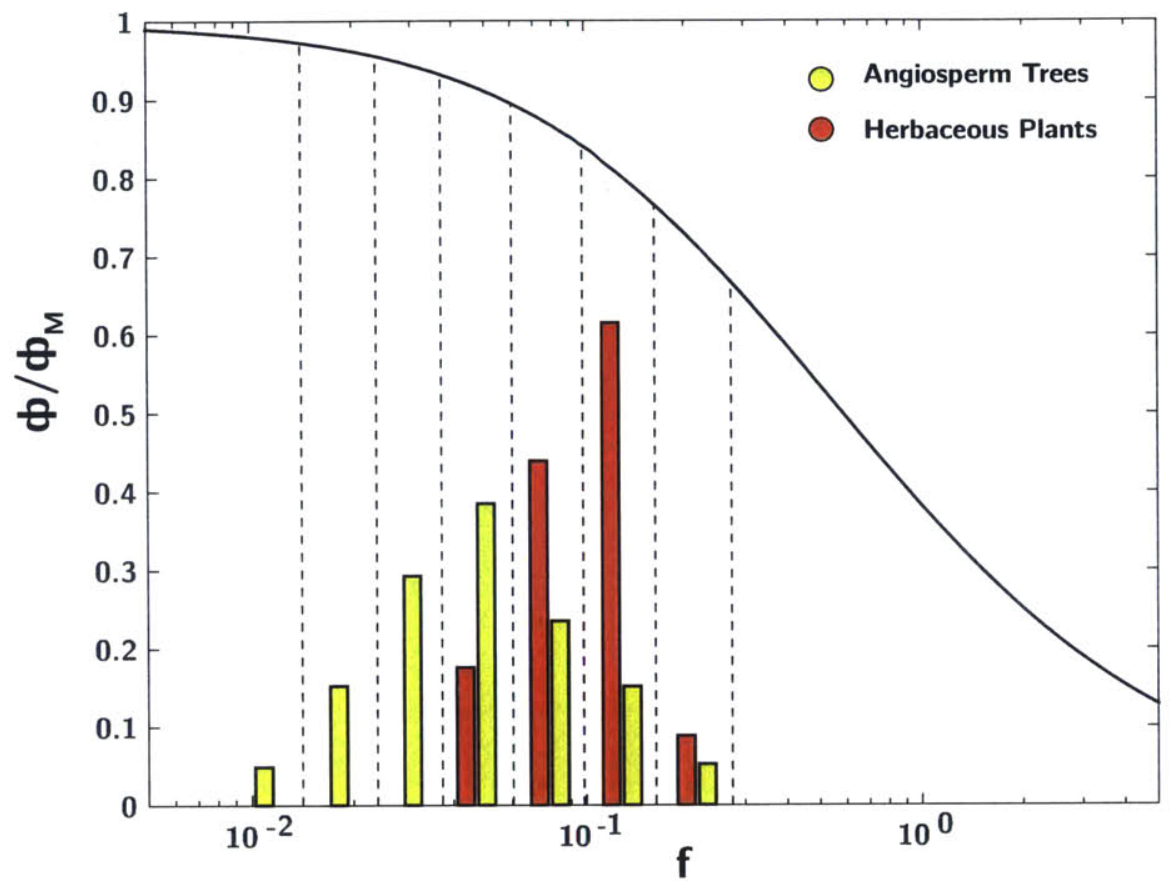

Figure 5-1: Increasing flushing numbers lead to a decrease in sugar export rate relative to the maximal export $\phi_{\mathrm{M}}=R T c_{0}^{2} / R_{\text {tot }}$. Histogram : estimation of the flushing number $L_{P}\left(R T c_{0}-\rho g h\right) / k_{\mathrm{D}}\left(1+R_{\mathrm{T}} / R_{\mathrm{M}}\right)^{-1}$ with $R_{\mathrm{T}}$ the transport phloem resistance and $R_{M}$ the loading phloem membrane resistance, $-\rho g h$ the xylem pressure decreasing with plant height $h$ and $R T c_{0}$ the osmotic pressure in the source, for a sample of Gymnosperm trees and herbaceous plants.

approximately constant with height, and does not exceed $20 \mu \mathrm{m}$, we estimate $f$ in those plants by taking a value $a=16.7 \mu \mathrm{m}$ for the sieve tube radius, corresponding to the average radius calculated from $N=17$ angiosperm trees [11].

Due to their large transport resistance and height, a significant proportion of trees are seen to operate in the optimal export regime, where the export rate is close to the optimal Münch export rate and where phloem concentration is very close to source concentration, in agreement with experimental observations [15] [19]. Passive phloem loading thus appears to represents an efficient way for sugar export in large trees. By contrast, most herbaceous plants, as well some of the smallest tree species are found to have a larger flushing number. This suggests that small herbaceous plants would not be able to take full advantage of passive phloem loading, because their large hydraulic 
permeabilities would prevent them from building up large sugar concentrations in the phloem, thus reducing phloem pressure. Note however that these conclusion must be taken with caution: although small passive loaders would be further from the optimal Münch regime, they would still export more than passive loaders of larger height (Fig. 3-2). Large plants should also be able to benefit from active loading by spending biochemical energy to increase phloem concentration. 


\section{Chapter 6}

\section{Discussion}

A relatively complete picture of the physical effects that influence transport in a passive phloem loader has emerged. We have explored the coupled dynamics between sugar loading by diffusion from a source, and osmotic convective export in a synthetic osmotic pump mimicking the passive phloem loading mechanism of sugars in plants. We have shown that this coupling can give rise to two distinct transport regimes, which are characterized by a flushing number, a system-scale Peclet number characterizing the relative importance of diffusion over convection in the system.

In the low flushing number regime, export is limited by the convection of water through the hydraulic circuit. This regime is the most favorable one in terms of export of water and sugars, as the concentration in the phloem is similar to that of the source. In the high flushing number regime, the solute in the phloem are evacuated by the convective flow of water they create, leading to a large concentration difference between the source and the phloem. The export is limited by the diffusion of sugars, as sugars are convectively exported as soon as they enter the phloem. An increase of the transport resistance in the diffusion limited regime leads to a build-up of sugar concentration in the phloem, and thus an increase of the driving force for long-distance flow through the sieve tubes. This regime thus leads to a weak scaling

of the flow rate with the system's resistance $\left(Q \sim R_{\text {tot }}^{-0.5}\right)$, which could be interesting in engineering applications where a constant export of water is needed regardless of the outlet pressure or resistance $R_{T}$. 
Due to the presence of a sugar source, our osmotic pump is able to provide sustained flow rate for several hours, and potentially to several days, depending on the size of the source and the solute reflectivity of the semipermeable membrane used, opening the door to new actuation mechanisms in microfluidic systems, drug delivery and microrobotics applications.

We then showed that the hydrostatic pressures which can be attained in our synthetic system are compatible with the pressure expected to drive translocation in large trees. We thus argue that active phloem loading is not required for long distance transport in plants, and that passive loaders can develop hydrostatic pressures as large as active loaders, but at the cost of large sugar concentrations in their leaf. Moreover, our experiments provide a direct experimental proof that large hydrostatic pressures can be osmotically generated.

The relevance of the flushing number could be assessed experimentally in passive loaders. An increase of the transport resistance, for example via the application of cold to inhibit long-distance transport on the epidermal tissues should lead to a direct build-up of phloem concentration. Fu et al. [4] showed that the few herbacious plants that load passively have reduced whole plant water conductivity. It would be interesting to see whether passive loading in herbacious plants also correlates with the specific macroscopic traits predicted by our analysis such as smaller sieve tube radii and enhanced plasmodesmatal permeabilities. The active loader arabidopsis with downregulated sucrose transporters was found to be able to complete its life cycle [16]. One could check whether phloem loading could occur symplastically in arabidopsis, in which plasmodesmata are present at all interfaces between the mesophyll and phloem and see whether the downhill concentration gradient between mesophyll and phloem is larger than in putative passive loaders.

Several explanations have been proposed to rationalize the use of different phloem loading strategies according to plant traits. In particular, Fu et al. [4] proposed that leaf cell water potential in tall trees would be imposed by low xylem tension. We showed here that the efficient regime of low flushing numbers is accessible to plants which load symplastically by diffusion through their plasmodesmata, provided the 
permeability of the plasmodesmatal interface is large enough, the transport resistance, proportional to plant height is large enough and the xylem pressure is small enough. Based on physiological data, we showed that most trees are thought to work in such regimes, where the concentration gradient between source cells and the phloem is small, as confirmed by experimental measurements. Our analysis also suggests that for similar permeabilities of the plasmodesmatal interface, if they were to load sugars passively by diffusion, herbaceous plants could see their solutes significantly flushed away by water convection because of their larger phloem hydraulic permeabilities, thus reducing the available driving force and phloem pressure. We thus argue that active phloem loading is not necessary to drive long distance transport in plants, and that efficient passive loading is more accessible to plants with large transport resistances like trees. Our analysis therefore provides a new and unexpected correlation between macroscopic plant traits and phloem loading strategies. 


\section{Appendix A}

\section{Solution to the coupled transport}

\section{equations}

We detail here the solution of the coupled transport equations and identify the flushing number as the relevant non-dimensional control parameter.

The two steady-state coupled equations for sugar and water balance are :

$$
\begin{aligned}
R_{\mathrm{tot}} Q_{W} & =P_{X}-P_{R}+R T c \\
Q_{W} \cdot c & =K_{D}\left(c_{0}-c\right)
\end{aligned}
$$

Eliminating $c$ in the equations above, we obtain a second order equation for $Q_{W}$ :

$$
Q_{W}^{2}+\left(K_{D}+\frac{P_{R}-\psi_{X}}{R_{\mathrm{tot}}}\right) Q_{W}-\frac{K_{D}}{R_{\mathrm{tot}}}\left(R T c_{0}+\psi_{X}-P_{R}\right)=0
$$

The solution is expressed as follow, with $\Delta P=P_{R}-\psi_{X}$

$$
Q_{W}=\frac{1}{2}\left(K_{D}+\frac{\Delta P}{R_{\mathrm{tot}}}\right)\left(\sqrt{1+\frac{4 K_{D} R_{\mathrm{tot}}\left(R T c_{0}-\Delta P\right)}{\left(K_{D} R_{\mathrm{tot}}+\Delta P\right)^{2}}}-1\right)
$$

Writing :

$$
f=\frac{R T c_{0}-\Delta P}{K_{D} R_{\mathrm{tot}}} \text { and } \tilde{\Delta} P=\frac{\Delta P}{K_{D} R_{\mathrm{tot}}}
$$


We obtain :

$$
\frac{Q_{W}}{K_{D}}=\frac{1}{2}(1+\tilde{\Delta} P)\left(\sqrt{1+\frac{4 f}{(1+\tilde{\Delta} P)^{2}}}-1\right)
$$

which, is the limit $\tilde{\Delta} P \ll 1$, gives :

$$
\frac{Q_{W}}{K_{D}}=\frac{1}{2}(\sqrt{1+4 f}-1)
$$

We obtain the following expressions for the dimensionless flow rates, concentration and sugar export rates, in the limit $\tilde{\Delta} P \ll 1$.

Flow Rate $Q_{W}\left[\mathrm{~m}^{3} / \mathrm{s}\right]$

$$
\begin{aligned}
\frac{Q_{W}}{K_{D}} & =\frac{1}{2}(\sqrt{1+4 f}-1) \\
\frac{Q_{W}}{Q_{W}^{\max }} & =\frac{\phi_{W} R_{\mathrm{tot}}}{R T c_{0}}=\frac{f}{2}(\sqrt{1+4 f}-1)
\end{aligned}
$$

Sugar Export $\dot{\phi}[\mathrm{mol} / \mathrm{s}]$

$$
\begin{aligned}
\frac{\dot{\phi}}{K_{D} c_{0}} & =\frac{\sqrt{1+4 f}-1}{\sqrt{1+4 f}+1} \\
\frac{\dot{\phi}}{\dot{\phi}_{\max }} & =\frac{\phi_{W} R_{\mathrm{tot}}}{R T c_{0}^{2}}=\frac{1}{f} \frac{\sqrt{1+4 f}-1}{\sqrt{1+4 f}+1}
\end{aligned}
$$

Concentration $c[\mathrm{~mol}]$

$$
\frac{c}{c_{0}}=\frac{2}{1+\sqrt{1+4 f}}
$$




\section{Appendix B}

\section{Evalutation of membrane resistances and dextran viscosity}

\section{Membrane Permeabilities}

To estimate the osmotic coefficient of dextran and the resistances of the semipermeable membranes, we inject sugar and dextran solutions of known concentration in the chamber on one side of the semipermeable membrane, pure water on the other side and measure the resulting flow rates.

We find linear relations between concentrations and flow rates, provided the flow through the membrane is small enough and does not induce solute depletion close to the membrane. We define membrane resistance for each solute $(s=\{$ suc, dex $\})$ as $R T c_{(s)}=R_{\mathrm{M}}^{(s)} Q$, were $Q$ is the flow of water $\left[\mathrm{m}^{3} / \mathrm{s}\right]$.

Because sucrose osmotic coefficient is unity, we can estimate the osmotic coefficient $\alpha$ of dextran as the ratio of membrane resistances of sucrose and dextran: $\alpha=$ $R_{M}^{(s u c)} / R_{M}^{(d e x)} \approx 4.7$

\section{Dextran Viscosity}

We measure the variation of dextran viscosity with concentration using a Cone and Plate rheometer. We approximate variation of dextran viscosity with concentration $c$ in $\mathrm{mmol} / \mathrm{L}$ as $\eta(c)=\eta_{w}\left(1+0.03334 \cdot c+0.0001384 \cdot c^{2}+1.079 \cdot 10^{-5} \cdot c^{3}\right)$ with $\eta_{w}=1$ cPs the viscosity of water. 


\section{Bibliography}

[1] Panadda Dechadilok and William M Deen. Hindrance factors for diffusion and convection in pores. Industrial \& Engineering Chemistry Research, 45(21):69536959, 2006.

[2] Henry H Dixon and John Joly. On the ascent of sap. Philosophical Transactions of the Royal Society of London. B, pages 563-576, 1895.

[3] Walter Eschrich, Ray F Evert, and John H Young. Solution flow in tubular semipermeable membranes. Planta, 107(4):279-300, 1972.

[4] Qiushi Fu, Lailiang Cheng, Yangdong Guo, and Robert Turgeon. Phloem loading strategies and water relations in trees and herbaceous plants. Plant Physiology, 157(3):1518-1527, 2011.

[5] Yuri Gamalei. Structure and function of leaf minor veins in trees and herbs. Trees, 3(2):96-110, 1989.

[6] Yuri Gamalei. Phloem loading and its development related to plant evolution from trees to herbs. Trees, 5(1):50-64, 1991.

[7] Kaare H Jensen, Emmanuelle Rio, Rasmus Hansen, Christophe Clanet, and Tomas Bohr. Osmotically driven pipe flows and their relation to sugar transport in plants. Journal of Fluid Mechanics, 636:371-396, 2009.

[8] Kaare H Jensen and Maciej A Zwieniecki. Physical limits to leaf size in tall trees. Physical review letters, 110(1):018104, 2013.

[9] Kåre Hartvig Jensen, J Lee, Tomas Bohr, Henrik Bruus, NM Holbrook, and MA Zwieniecki. Optimality of the münch mechanism for translocation of sugars in plants. Journal of the Royal Society Interface, 8(61):1155-1165, 2011.

[10] Kåre Hartvig Jensen, Jinkee Lee, Tomas Bohr, and Henrik Bruus. Osmotically driven flows in microchannels separated by a semipermeable membrane. Lab on a chip, 9(14):2093-2099, 2009.

[11] Kåre Hartvig Jensen, Johannes Liesche, Tomas Bohr, and Alexander Schulz. Universality of phloem transport in seed plants. Plant, cell $\&$ environment, 35(6):1065-1076, 2012. 
[12] Erik Kjeang. Microfluidic Fuel Cells and Batteries. Springer, 2014.

[13] ALEXANDER LANG. A working model of a sieve tube. Journal of Experimental Botany, 24(5):896-904, 1973.

[14] Ernst Münch. Die stoffbewegungen in der pflanze. Jena: Fischer, 1930.

[15] William A Russin and Ray F Evert. Studies on the leaf of populus deltoides (salicaceae): ultrastructure, plasmodesmatal frequency, and solute concentrations. American journal of botany, pages 1232-1247, 1985.

[16] Avinash C Srivastava, Kasturi Dasgupta, Eric Ajieren, Gabriella Costilla, Roisin C McGarry, and Brian G Ayre. Arabidopsis plants harbouring a mutation in atsuc2, encoding the predominant sucrose/proton symporter necessary for efficient phloem transport, are able to complete their life cycle and produce viable seed. Annals of botany, page mcp215, 2009.

[17] Abraham D Stroock, Vinay V Pagay, Maciej A Zwieniecki, and N Michele Holbrook. The physicochemical hydrodynamics of vascular plants. Annual Review of Fluid Mechanics, 46:615-642, 2014.

[18] Robert Turgeon. The puzzle of phloem pressure. Plant physiology, 154(2):578$581,2010$.

[19] Robert Turgeon and Richard Medville. The absence of phloem loading in willow leaves. Proceedings of the National Academy of Sciences, 95(20):12055-12060, 1998.

[20] Tobias D Wheeler and Abraham D Stroock. The transpiration of water at negative pressures in a synthetic tree. Nature, 455(7210):208-212, 2008.

[21] Cankui Zhang, Lu Han, Thomas L Slewinski, Jianlei Sun, Jing Zhang, Zeng$\mathrm{Yu}$ Wang, and Robert Turgeon. Symplastic phloem loading in poplar. Plant physiology, 166(1):306-313, 2014. 